Supporting Information

\title{
Revealing the Dynamic Mechanism by Which Transferrin Promotes the Cellular Uptake of HAIYPRH Peptide-Conjugated Nanostructures by Force Tracing
}

Siying Li ${ }^{\dagger}$, , Ruixia Wang ${ }^{\dagger}$, Junfeng Li $i^{\dagger}$, Yulin Liu ${ }^{\dagger}$, Yanfeng $F u^{\dagger}$, Jing Zhou ${ }^{\dagger}$, Guocheng Yang ${ }^{\dagger}$ \& Yuping Shan ${ }^{\dagger}, *$

${ }^{\dagger}$ School of Chemistry and Life Science, Advanced Institute of Materials Science, Changchun University of Technology, Changchun 130012, China.

${ }^{\S}$ School of Chemical Engineering, Changchun University of Technology, Changchun 130012, China.

Corresponding Author

Yuping Shan, School of Chemistry and Life Science, Advanced Institute of Materials

Science, Changchun University of Technology, Changchun 130012, China; Email:

shanyp@ciac.ac.cn 


\section{Material and methods}

Materials

Holo-Tf was purchased from Sigma Aldrich (St. Louis, MO). T7 with a cysteine on the N-terminal (cys-T7) was purchased from Chinese Peptide Company (Hangzhou, China). $50 \mathrm{~nm}$ AuNPs were synthesized by Nanjing JCNANO Technology Co., Ltd. Holo-Tf-Cy5 was pur-chased from Xi'an Qiyue Biotechnology Co., Ltd.

Cell culture

The lung cancer cells (A549), african green monkey kidney cells (Vero), and human cervical cancer cells (HeLa) were all purchased from the Institutes of Biological Sciences (Shanghai, China). A549 and HeLa cells were cultured in supplemented with Dulbecco's modified Eagle medium (DMEM, BI), and Vero cells were cultured in Minimum Eagle Medium (MEM, BI). Each type of medium was supplemented with $10 \%$ fetal bovine serum, penicillin $(100 \mu \mathrm{g} / \mathrm{mL})$, and streptomycin $(100 \mu \mathrm{g} / \mathrm{mL})$, and the cells were maintained at $37{ }^{\circ} \mathrm{C}$ in a humidified incubator containing $5 \% \mathrm{CO}_{2}$. The cells were subcultured for $24-36$ h until $75 \%$ of the petri dish was covered by cells. The cells were washed with PBS (phosphate buffer solution, $137 \mathrm{mM} \mathrm{NaCl}, 2 \mathrm{mM}$ $\mathrm{KCl}, 8 \mathrm{mM} \mathrm{Na} \mathrm{HPO}_{4}, 1.5 \mathrm{mM} \mathrm{KH} \mathrm{PO}_{4}, \mathrm{pH}$ 7.4) for three times and serum-free medium (SFM) one time in sequence to remove cell debris and unattached cells before using in force tracing experiments. The force tracing tests were carried out at $37^{\circ} \mathrm{C} .{ }^{1}$ 
Synthesis and characterization of AuNP-T7

AuNPs were reacted with cys-T7 at the molar ratio of 1:5, and stirred at room temperature for $24 \mathrm{~h}$ to synthesize AuNP-T7 according the previous report. ${ }^{2}$ The resulting conjugates were purified through a $3 \mathrm{kD}$ Molecular Weight Cutoff (MWCO) microcon centrifugal filter device to remove free $\mathrm{T} 7$. The particle size and zeta potential of AuNP-T7 was measured using dynamic light scattering (DLS). The prepared AuNP-T7 was analyzed by UV-vis absorption spectroscopy. The morphology of AuNPs-T7 was assessed with the transmission electron microscopy, nanoparticle solution was dried on a carbon film grid.

\section{AFM tip modification}

AuNP-T7 was functionalized onto AFM tip (MSCT, Santa Barbara, CA) as previously described. ${ }^{3}$ In brief, the AFM tips were firstly functionalized with 3-aminopropyltriethoxysilane (APTES). The desiccator was purged with argon for 3 min, and $50 \mu \mathrm{L}$ of APTES (99\%) and $20 \mu \mathrm{L}$ of N,N-diisopropylethylamine (99\%) were placed into a small container at the bottom of the desiccator, respectively, leaving the AFM tips exposed to APTES vapor for $2 \mathrm{~h}$. Subsequently, the PEG cross-linker (SH-PEG 76 -NHS, MW: 3400) was attached to the APTES modified AFM tips with presence of triethylamine and trichloromethane. At last, The AFM tips were immersed in $200 \mu \mathrm{L}$ solution containing AuNP-T7 for $1 \mathrm{~h}$, after functionalization, the AFM tips were washed with water for three times and protect from light stored at $4{ }^{\circ} \mathrm{C}$ in water until use. The AuNP-T7 modified AFM tip was characterized using 
scanning electron microscope (JEOL JSM-7610F).

Force tracing measurements

The force tracing measurements were performed on AFM 5500 (Agilent Technologies, Chandler, AZ). The experiments were carried out at $37^{\circ} \mathrm{C}$ controlled by temperature controller 325 (Agilent Technologies, Chandler, AZ). With the help of a CCD camera, the AuNP-T7 modified AFM tip was located onto the cell membrane. The force-distance curves were obtained to find out the contact point between the AuNP-T7 modified AFM tip and the cell membrane. When the AFM tip tethered with AuNP-T7 contacted with cell membranes, the feedback system was turned off and the movement of the AFM tip was stopped. ${ }^{4}$ The deflection of the cantilever caused by the internalization of AuNP-T7 was recorded by a 16-bit DA/AD card (PCI-6361e, National Instruments, US), which was controlled by LabVIEW software. During the experiments, thousands of force tracing curves were collected at different cells and analyzed by LabVIEW (National Instruments Inc., Austin, Texas, USA). The sensitivity and the spring constant of the AFM tip were determined according to previous report. $^{5}$

Blocking experiments

In blocking experiments, A549 cells were coincubated with $\mathrm{Tf}$ (final concentration of $25 \mu \mathrm{M}$ ) and AuNP-T7 (final concentration of $50 \mathrm{pM}$ ) for $10 \mathrm{~min}, 20 \mathrm{~min}$, and $1 \mathrm{~h}$, 
ferristatin (final concentration of $50 \mu \mathrm{M})$ for $1 \mathrm{~h}(+$ Ferristatin $1 \mathrm{~h})$ and $4 \mathrm{~h}(+$ Ferristatin 4 h), respectively.

Calculating the average speed of AuNP-T7 entry cell

The average speed during the process of AuNP-T7 entry cell was calculated by $D /$ duration. The displacement $D$ of AuNP-T7 consists of the bending distance of the AFM tip cantilever $d$ and the extension length of the PEG linker $h$ (Figure S7).

$$
D=d+h(1)
$$

The following formula is Worm-like-Chain (WLC) model, explaining the relationship between PEG stretching length and the force. ${ }^{6}$

$$
\frac{F L_{p}}{K_{B} T}=\frac{1}{4}\left(1-\frac{h}{L_{0}}+\frac{F}{K_{0}}\right)^{-2}-\frac{1}{4}+\frac{h}{L_{0}}-\frac{F}{K_{0}}
$$

Where $L_{p}$ represents the persistence length, $k_{B}$ stands for the Boltzmann constant, $T$ is the absolute temperature, $L_{0}$ is the contour length, $h$ represents the extension of PEG linker, $k_{0}$ is the enthalpic correction, which is $1561 \pm 33 \mathrm{pN}$, the length of PEG unit is $4.2 \AA$, the total estimated contour length $L_{0}$ for PEG of $76-77$ is nearly $321 \AA{ }^{3}$ The bending distance $d$ of the AFM tip cantilever could be obtained by Hooke's law:

$$
F=k \times d(3)
$$

Where $F$ is the force measured from the force tracing curves of AuNP-T7 entry cell, and $k$ represents the spring constant of the AFM tip cantilever. The displacement $D$ could be obtained from the above formulas (1-3), as shown in Table S2.

Fluorescence imaging 
A549 cells, HeLa cells, and Vero cells were subcultured for $24-36$ h until $75 \%$ of the petri dish was covered by cells. Holo-Tf-Cy5 (final concentration of $1.73 \mu \mathrm{M}$ ) was coincubated with cells in SFM for $1 \mathrm{~h}$ at $37{ }^{\circ} \mathrm{C}$. The cells were washed with PBS for three times in advance of fluorescence imaging. Fluorescence imaging was performed on a fluorescence microscope (Nikon-Ti-S). Cy5 was excited with a $625 \mathrm{~nm} \mathrm{He}-\mathrm{Ne}$ laser. The fluorescence intensity is calculated using the software ImageJ.

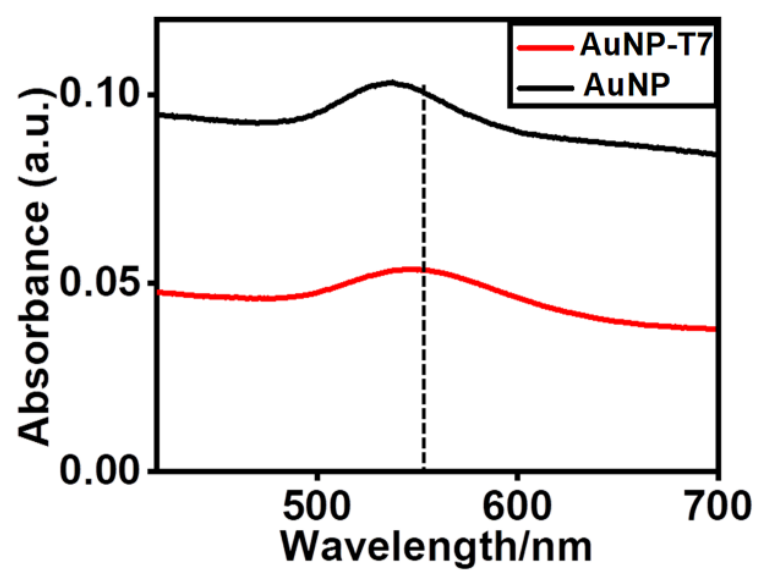

Figure S1. UV-vis spectra of AuNPs (black) and AuNPs-T7 (red). A red shift about $10 \mathrm{~nm}$ from $534 \mathrm{~nm}$ (black) to $544 \mathrm{~nm}$ (red) upon peptide modification, indicating the conjugation of $\mathrm{T} 7$ on the AuNP. 


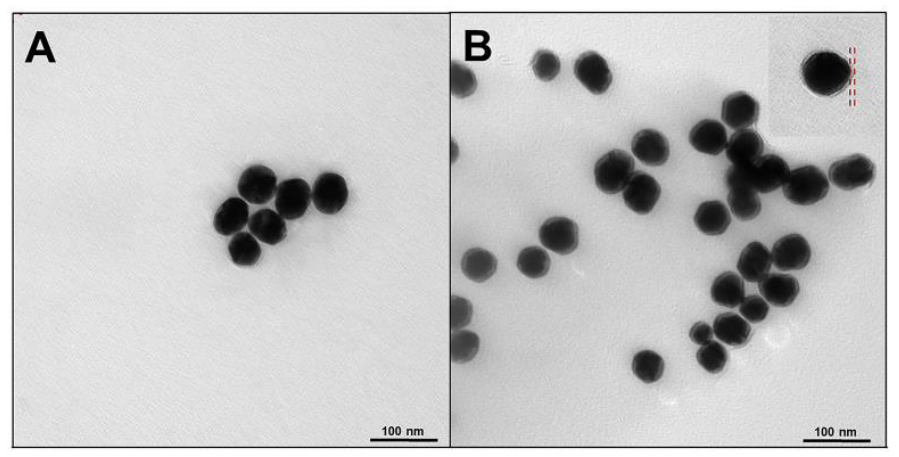

Figure S2. TEM images of AuNP and AuNP-T7. (A) The TEM image of AuNPs. (B) The TEM image of AuNPs-T7. The illustration in the upper right corner is an enlarged view, and the thin layer between the two red dotted lines indicates that T7 hs been covered on AuNP. Scale bar is $100 \mathrm{~nm}$. TEM images indicate that most AuNPs are uniform in size, and after coating with T7, a characteristic "peptide corona" could be seen, indicating that the T7 coating does not alter the morphology of the AuNPs.

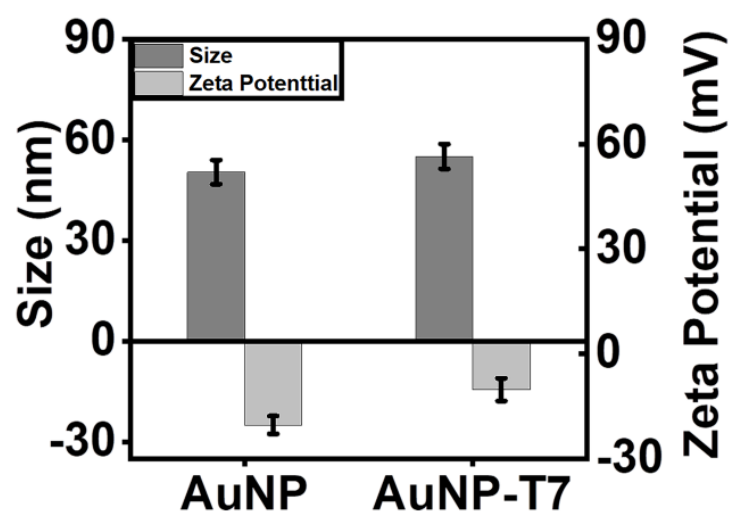

Figure S3. The size and zeta potential distribution of the AuNPs and AuNPs-T7. 


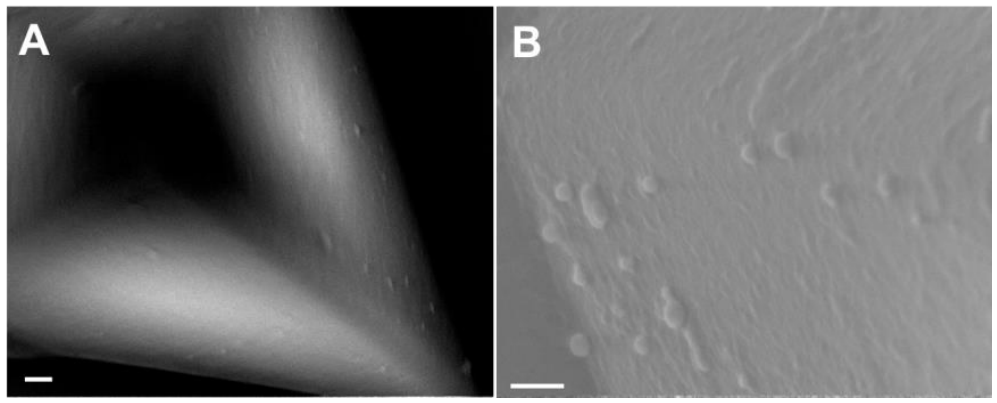

Figure S4. SEM image of AuNP-T7-modified AFM tip. (A) The SEM image of the AFM tip. Scale bar is $200 \mathrm{~nm}$. (B) Side wall of the AFM tip. Scale bar is $100 \mathrm{~nm}$.

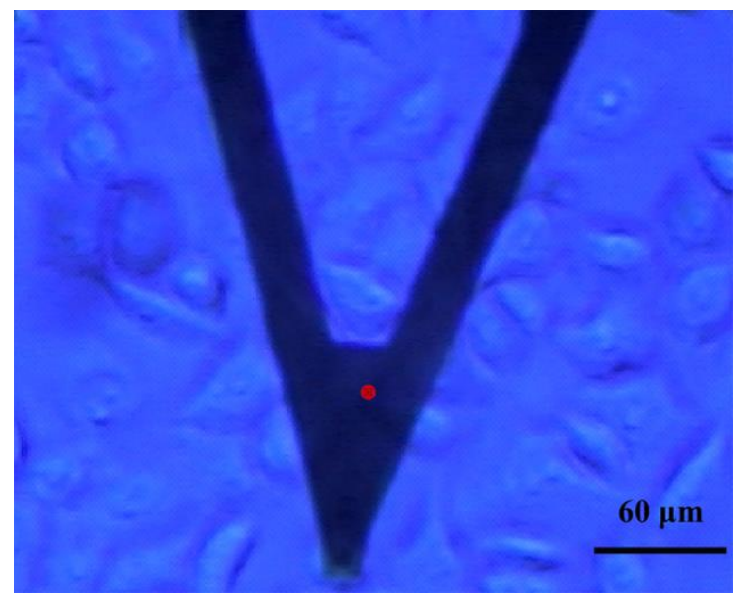

Figure S5. CCD camera is used to locate the AFM tip above the cell.

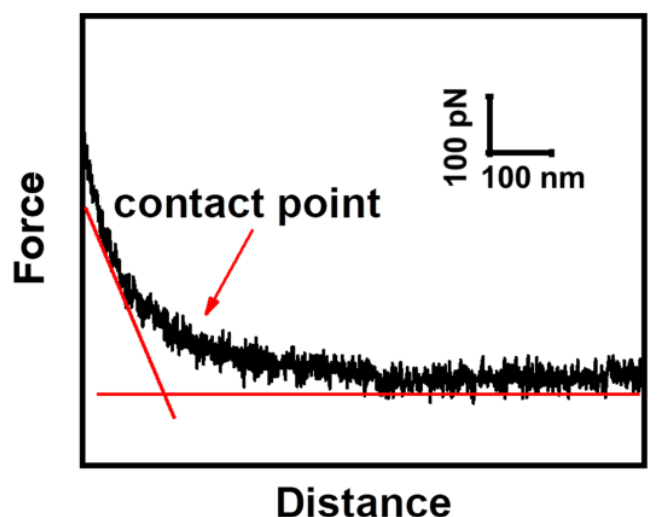

Figure S6. The contact point between the AuNP-T7 modified AFM tip and the cell surface. The contact point is the intersection of the slope and flat part in the force-distance curve. 


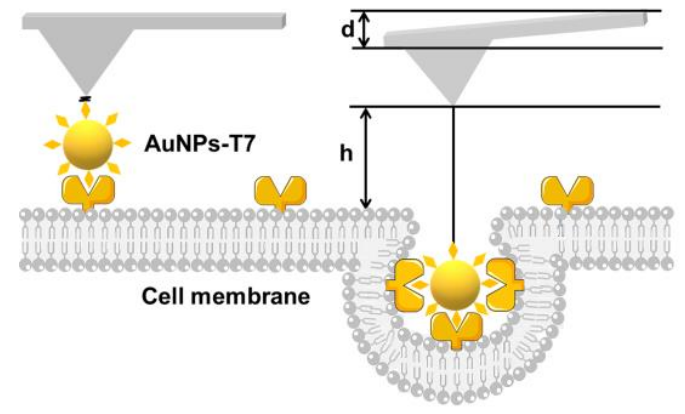

Figure S7. Schematic of displacement during the process of AuNP-T7 entry cell. The displacement consists of the bending distance $d$ of the AFM tip cantilever and the extension length $h$ of the PEG linker.

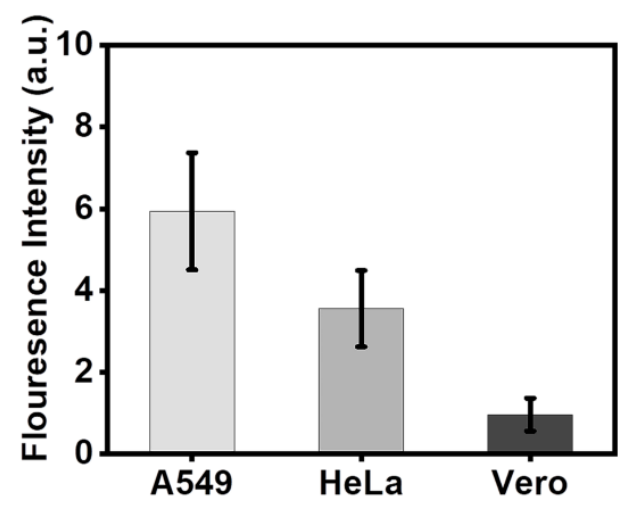

Figure S8. Fluorescence intensity of A549, HeLa and Vero cells after coincubation with Holo-Tf-Cy5 for $1 \mathrm{~h}$. 
Table 1. Dynamic parameters of cellular uptake of AuNP-T7 in different cell lines that coincubated with SFM for $1 \mathrm{~h}$ and coincubated with free Tf for $1 \mathrm{~h}$.

\begin{tabular}{|c|c|c|c|c|c|c|c|c|}
\hline \multirow[b]{2}{*}{ Cell } & \multicolumn{4}{|c|}{ Coincubation with SFM for $1 \mathrm{~h}$} & \multicolumn{4}{|c|}{ Coincubation with free $\mathrm{Tf}$ for $1 \mathrm{~h}$} \\
\hline & Speed & Force & Time & Probabiliy & Speed & Force & Time & Probability \\
\hline line & $(\mu \mathrm{m} / \mathrm{s})$ & $(\mathrm{pN})$ & (ms) & $(\%)$ & $(\mu \mathrm{m} / \mathrm{s})$ & $(\mathrm{pN})$ & (ms) & $(\%)$ \\
\hline A549 & 0.396 & $66.70 \pm 22.86$ & $85.00 \pm 30.13$ & 8.26 & 0.496 & $53.49 \pm 16.83$ & $67.24 \pm 27.87$ & 12.76 \\
\hline $\mathrm{HeLa}$ & 0.332 & $62.58 \pm 22.41$ & $100.93 \pm 45.55$ & 7.96 & 0.410 & $59.00 \pm 36.81$ & $81.53 \pm 39.80$ & 9.22 \\
\hline Vero & 0.302 & $74.69 \pm 34.10$ & $112.06 \pm 62.30$ & 2.18 & 0.227 & $69.17 \pm 25.46$ & $148.59 \pm 11.96$ & 2.22 \\
\hline
\end{tabular}

Table 2 The displacement $D$ for cellular uptake of AuNP-T7 in different cell lines that coincubated with SFM for $1 \mathrm{~h}$ and coincubated with free Tf for $1 \mathrm{~h}$.

Coincubation with SFM for $1 \mathrm{~h} \quad$ Coincubation with free $\mathrm{Tf}$ for $1 \mathrm{~h}$

$\begin{array}{lrc}\text { Cell line } & D(\mathrm{~nm}) & D(\mathrm{~nm}) \\ \text { A549 } & 33.66 & 33.35 \\ \text { HeLa } & 33.51 & 33.43 \\ \text { Vero } & 33.84 & 33.73\end{array}$

SFM: serum-free medium. 


\section{REFERENCES}

(1) Yang, B.; Xu, H.; Wang, S.; Cai, M.; Shi, Y.; Yang, G.; Wang, H.; Shan, Y.

Studying the Dynamic Mechanism of Transporting a Single Drug Carrier-Polyamidoamine Dendrimer Through Cell Membranes by Force Tracing. Nanoscale. 2016, 8, 18027-18031.

(2) Zong, J.; Cobb, S. L.; Cameron, N. R. Peptide-Functionalized Gold Nanoparticles: Versatile Biomaterials for Diagnostic and Therapeutic. Applications. Biomater Sci. 2017, 5, 872-886.

(3) Yang, Y.; Zhang, Q.; Cai, M.; Xu, H.; Lu, D.; Liu, Y.; Fu, Y.; Yang, G.; Shan, Y. Size-Dependent Transmembrane Transport of Gold Nanocages. ACS Omega. 2020, 5, 9864-9869.

(4) Lu, D.; Yang, X.; Zhang, Q.; Wang, R.; Zhou, S.; Yang, G.; Shan, Y. Tracking the Single-Carbon-Dot Transmembrane Transport by Force Tracing Based on Atomic Force Microscopy. ACS Biomater Sci Eng. 2018, 5, 432-437.

(5) Shan, Y.; Ma, S.; Nie, L.; Shang, X.; Hao, X.; Tang, Z.; Wang, H. Size-Dependent Endocytosis of Single Gold Nanoparticles. Chem Commun (Camb). 2011, 47, 8091-8093.

(6) Kienberger, F.; Pastushenko, V. P.; Kada, G.; Gruber, H. J.; Riener, C.; Schindler, H.; Hinterdorfer, P. Static and Dynamical Properties of Single Poly(Ethylene Glycol) Molecules Investigated by Force Spectroscopy. Single Mol. 2000, 1, 123-128. 